\title{
1 Nuclear soluble cGAS senses DNA virus infection
}

2

3 Yakun Wu ${ }^{1 \dagger}$, Kun Song ${ }^{1 \dagger}$, Wenzhuo Hao ${ }^{1}$, Lingyan Wang ${ }^{1}$, Shitao $\mathrm{Li}^{{ }^{*}}$

4

$5{ }^{1}$ Department of Microbiology and Immunology, Tulane University, New Orleans, LA 70118,

6 USA

7

$8{ }^{\dagger}$ These authors have contributed equally to this work.

9

10 *To whom correspondence should be addressed. Tel: 01-504-988-2203; Fax: 504-988-5144;

11 Email: sli38@tulane.edu.

12

13 Running Title: Leaked chromatin-bound cGAS senses viral infection

14

15 Keywords: Innate immunity, DNA sensing, cGAS, chromatin, interferon, HSV-1

16 


\section{ABSTRACT}

18 The cytosolic DNA sensor cGAS detects foreign DNA from pathogens or self-DNA from cellular

19 damage and instigates type I interferon (IFN) expression. Recent studies find that cGAS also

20 localizes in the nucleus and binds the chromatin. Despite how cGAS is inhibited in the nucleus

21 is well elucidated, whether nuclear cGAS participates in DNA sensing is not clear. Here, we

22 report that herpes simplex virus 1 (HSV-1) infection caused the release of cGAS from the

23 chromatin into the nuclear soluble fraction. Like its cytosolic counterpart, the leaked nuclear

24 soluble cGAS could sense viral DNA, produce cGAMP, and induce mRNA expression of type I

25 IFN and interferon-stimulated genes. Furthermore, the nuclear cGAS limited HSV-1 infection.

26 Taken together, our study demonstrates that HSV-1 infection releases cGAS from the chromatin

27 tethering and, in turn, the nuclear soluble cGAS activates type I IFN production. 


\section{INTRODUCTION}

29 Cytosolic DNA from infectious microbe triggers innate host defense by activating type I IFN 30 expression (Barber, 2015; Chen et al., 2016; Hornung et al., 2014; Zevini et al., 2017). Cytosolic

31 DNA is recognized by the recently identified DNA sensor, cyclic GMP-AMP synthase (cGAS,

32 also known as MB21D1) (Sun et al., 2013). After binding to DNA, cGAS produces cyclic GMP-

33 AMP (cGAMP) (Sun et al., 2013). cGAMP is a second messenger that binds to the endoplasmic

34 reticulum membrane protein, stimulator of interferon genes (STING, also known as TMEM173,

35 MPYS, MITA, and ERIS), leading to STING dimerization (Ishikawa and Barber, 2008; Sun et al.,

36 2009; Zhang et al., 2013; Zhong et al., 2008). Subsequently, STING recruits TANK-binding

37 kinase 1 (TBK1) to the endoplasmic reticulum and activates TBK1. Activated TBK1

38 phosphorylates interferon regulatory factors (IRFs), which triggers the dimerization and nuclear

39 translocation of IRFs. In the nucleus, IRFs form active transcriptional complexes and activate

40 type I IFN gene expression (Fitzgerald et al., 2003; Hemmi et al., 2004; McWhirter et al., 2004;

41 Sharma et al., 2003; Tanaka and Chen, 2012).

42

43 Excessive host DNA activates the cGAS signaling pathway, leading to aberrant IFN activation

44 and autoimmune diseases, such as Aicardi-Goutieres syndrome (AGS) (Pokatayev et al., 2016).

45 Therefore, cells must render cGAS inert to host genomic DNA, and paradoxically, at the same

46 time, cells need to keep cGAS agile to foreign DNA. The old paradigm is that host DNA is

47 normally restricted to cellular compartments, such as the nucleus and the mitochondria. As

48 cGAS was first thought to be a solely cytosolic protein, the physical barrier blocks the access of

49 cGAS to host DNA (Cai et al., 2014; Chen et al., 2016). However, recent studies further found

50 other subcellular localizations of cGAS, including predominantly in the nucleus (Volkman, 2019),

51 on the plasma membrane (Barnett et al., 2019), mitosis-associated nuclear localization (Gentili

52 et al., 2019; Yang et al., 2017), or phosphorylation-mediated cytosolic retention (Liu et al., 
53 2018). Nonetheless, the nuclear localization of cGAS has been validated by many independent

54 laboratories (Gentili et al., 2019; Mackenzie et al., 2017; Volkman, 2019), which is of particular

55 interest as the nucleus is a DNA-rich environment. Nuclear cGAS is bound to the chromatin in

56 the nucleus by binding to the $\mathrm{H} 2 \mathrm{~A}-\mathrm{H} 2 \mathrm{~B}$ dimer of the nucleosome, which immobilizes cGAS on

57 the chromatin; thus, cGAS cannot access the nearby DNA to form an active dimer (Boyer et al.,

58 2020; Cao et al., 2020; Kujirai et al., 2020; Michalski et al., 2020; Pathare et al., 2020; Zhao et

59 al., 2020). In addition, the phosphorylation of the N-terminus of cGAS and the S291 site at the

60 C-terminus further prevents cGAS from activation during mitosis (Li et al., 2021; Zhong et al.,

61 2020). Although the mechanism of nuclear cGAS inhibition is well elucidated, the role of nuclear

62 cGAS in DNA sensing is unknown.

64 Recent studies showed that the murine R222 (R236 in human cGAS) and murine R241 (R255

65 in human) sites are the critical sites for the binding to the H2A-H2B dimer (Boyer et al., 2020;

66 Kujirai et al., 2020; Michalski et al., 2020; Pathare et al., 2020; Volkman, 2019; Zhao et al.,

67 2020). The mutation of these two arginines to glutamic acids leads to disruption of the binding to

68 the chromatin and the release of cGAS to nuclear soluble fraction, resulting in cGAS activation

69 and cGAMP production (Volkman, 2019). However, whether and how chromatin-bound cGAS is

70 released into nuclear soluble fraction under physiological or pathological conditions is not clear.

71

72 Here, we found that endogenous cGAS tethered with the chromatin in multiple cell lines and

73 HSV-1 infection caused the release of cGAS from the chromatin into the nuclear soluble

74 fraction. The nuclear soluble cGAS bound viral DNA and produced cGAMP. Furthermore, cells

75 exclusively expressing nuclear cGAS responded to HSV-1 infection and activated type I IFN

76 expression. Collectively, our study suggests that HSV-1 infection leads to cGAS release from

77 the chromatin tethering; in turn, the nuclear soluble cGAS senses viral DNA and activates type I 
78 IFN to suppress viral infection. Our study uncovers the role of nuclear cGAS in host defense to

79 DNA virus infection.

\section{RESULTS}

\section{Endogenous cGAS localizes in the cytoplasm and the nucleus.}

83 To determine the size of endogenous cGAS protein complex, we performed a sucrose gradient 84 ultracentrifugation for cell lysates from RAW 264.7 macrophages. Unexpectedly, the majority of 85 cGAS proteins were distributed in the high molecular weight fractions with a high sedimentation 86 rate (Fig. 1A). Interestingly, histone $\mathrm{H} 3$ co-fractionated with cGAS in most fractions with high 87 molecular weight (Fig. 1A), suggesting that cGAS might associate with the chromatin (Volkman, 88 2019). To further determine the subcellular localization of endogenous cGAS, we performed 89 subcellular fractionations in multiple cell lines. We fractionated the cell lysates into five fractions:

90 cytosol, membrane, nuclear soluble, chromatin-bound, and cytoskeletal. The nuclear soluble

91 fraction is extracted in a low salt concentration and does not contain histones and nucleosomes,

92 whereas the chromatin-bound fraction comprises nucleosomes with a high salt concentration

93 extract condition. We first fractionated H1299 cell lysates. cGAS was found in the cytosol and 94 the nucleus of H1299 cells (Fig. 1B). Consistent with a previous report (Volkman, 2019), cGAS 95 localized in the chromatin-bound nuclear fraction but not the nuclear soluble fraction (Fig. 1B).

96 Similar results were observed in THP-1 cells (Fig. S1A). We also examined whether DNA 97 stimulation altered cGAS distribution in each fraction. However, the amount of cGAS in each 98 fraction after stimulation was comparable to the fraction without DNA stimulation (Figs. 1B and $99 \mathrm{~S} 1 \mathrm{~A})$.

100

101 Next, we examined endogenous cGAS localization in cells by immunofluorescence assays 102 (IFA). We used a newly developed anti-human cGAS antibody by Cell Signaling Technology 103 and validated it by western blotting (Fig. S1B) and IFA (Fig. 1C) in cGAS wild type and knockout 
$104 \mathrm{H} 1299$ cells. Using this antibody, we found that cGAS localized in the cytosol, nucleus, nucleoli,

105 micronuclei, chromosome, chromatin bridge, and perinuclear region (Fig. 1D). Furthermore, we

106 examined the endogenous cGAS localization in several other cell lines. However, the patterns

107 of endogenous cGAS distribution varied in different cell lines, irrespective of cytosolic DNA

108 stimulation (Figs. 1C, S1C-S1F). In agreement with previous studies, we found that endogenous

109 cGAS localized in the nucleus in all tested cell lines, suggesting a potential role of nuclear 110 cGAS.

111

112 The $\mathrm{N}$-terminus and NES regulate cGAS nuclear localization.

113 Previous efforts have been made to determine the region responsible for cGAS nuclear 114 localization. One study suggested that the region of amino acids 161-212 is essential for 115 cytosolic retention of human cGAS (Gentili et al., 2019). There is a conserved classic nuclear

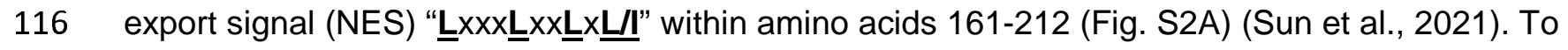
117 examine the role of the NES in cGAS subcellular localization, we deleted the NES in cGAS. IFA 118 assays showed that the NES deletion led to a slight increase of nuclear cGAS (Fig. S2B). We 119 further mutated two leucines in the NES into arginine and lysine (LL/RK) in mouse cGAS, 120 respectively. These mutations not only disrupt the NES but also converted the NES into a 121 nuclear localization signal (NLS) (Fig. 1E). IFA assays showed that the LL/RK mutation 122 dramatically increased cGAS nuclear localization (Fig. 1F). However, there were still 123 approximately $37 \%$ of cells in which cGAS resided in both the nucleus and the cytoplasm (Fig.

124 1F). These data suggest that the NES might be required but not sufficient for cGAS cytosolic 125 localization.

127 We further examined which domain might control cGAS nuclear localization (Fig. S2A). We 128 stably transfected the $\mathrm{N}$-terminal domain $(\mathrm{N})$ and the $\mathrm{N}$ deletion mutant (deIN) of cGAS into 129 HEK293 cells. Subcellular fractionation found that the $\mathrm{N}$ and delN showed distinct localizations 
130 (Fig. S2C). The $\mathrm{N}$ was mainly distributed in the cytosol with a small portion in the nuclear

131 soluble fraction. As reported recently ( $\mathrm{Li}$ et al., 2021), the delN localized in the membrane

132 fraction due to the exposure of mitochondrial targeting signal (MTS) (Fig. S2A). Furthermore,

133 delN was also found in the chromatin-bound and cytoskeletal fractions (Fig. S2C). IFA assays

134 further corroborated the cytosolic localization of the $\mathrm{N}$ and the mitochondrial and nuclear 135 localizations of deIN (Fig. S2D), suggesting that the $\mathrm{N}$-terminus is also involved in cGAS

136 cytosolic localization. Thus, we mutated the two leucines in the NES into arginine and lysine in

137 deIN of cGAS (deIN-LL/RK) to disrupt the NES and MTS. Interestingly, subcellular fractionation

138 found that the deIN-LL/RK mutant is exclusively expressed in the chromatin-bound fraction (Fig.

139 1G). Like endogenous cGAS, cytosolic DNA stimulation had little effect on the localization of

140 deIN-LL/RK (Fig. 1G). Overall, our data suggest that both NES and the N-terminal domain

141 regulate cGAS subcellular localization.

HSV-1 infection causes cGAS release from the chromatin to the nuclear soluble fraction.

144 As most DNA viruses are uncoated and then replicate in the nucleus, it would be the best

145 opportunity for the host to detect viral DNA in the nucleus at the early stage of viral infection.

146 However, nuclear cGAS is immobilized on the chromatin. We hypothesized that DNA virus

147 infection in the nucleus might cause cGAS release from the chromatin. In this regard, we

148 infected RAW 264.7 cells with herpes simplex virus type 1 (HSV-1), a DNA virus that replicates

149 in the nucleus. As shown in Figure 2A, HSV-1 infection caused a portion of cGAS to translocate

150 to the nuclear soluble fraction. By contrast, cytosolic DNA stimulation has little effect on cGAS

151 subcellular localization in RAW 264.7 macrophages (Fig. S3A). Furthermore, we also examined

152 three other viruses, vaccinia virus (VACV), influenza A viruses (IAV), and vesicular stomatitis

153 virus (VSV). VACV is a DNA virus that replicates in the cytoplasm. IAV and VSV are RNA

154 viruses and replicate in the nucleus and the cytosol, respectively. However, all these viruses

155 failed to induce cGAS translocation to the nuclear soluble fraction in RAW 264.7 macrophages 
156 (Figs. S3B-S3D), suggesting that DNA virus replication in the nucleus is required for cGAS

157 release from the chromatin to the nuclear soluble fraction.

\section{Inhibiting HSV-1 replication blocks cGAS release from the chromatin.}

160 To determine the mechanism by which HSV-1 induces cGAS release from the chromatin, we

161 first examined the effects of HSV-1 d109 mutant virus in which the IFN-suppression viral genes

162 are deleted (Eidson et al., 2002). As shown in Figure 2B, the d109 mutant also induced nuclear

163 soluble cGAS, suggesting these IFN-suppression viral proteins have little effect on cGAS

164 localization. Next, we examined the effects of viral replication on cGAS release. The HSV-1

165 DNA polymerase inhibitor acyclovir was used to inhibit viral replication. As predicted, viral 166 protein ICP8 expression reduced after acyclovir treatment. More interestingly, acyclovir blocked 167 viral infection-induced nuclear soluble cGAS (Fig. 2C).

As HSV-1 infection can cause nuclear stress and host DNA damage, we suspected that DNA damage might induce the release of cGAS from chromatin tethering. In this regard, we treated

171 RAW 264.7 macrophages with a DNA damage agent, cisplatin (Hu et al., 2016). As shown in

172 Figure 2D, cisplatin induced $\square \gamma \mathrm{H} 2 \mathrm{AX}$ (phosphorylated S139 H2AX histone), a hallmark of DNA

173 damage. However, cisplatin failed to induce nuclear soluble cGAS (Fig. 2D). Taken together,

174 these data suggest that DNA virus nuclear replication, but not IFN-suppression viral proteins or

175 DNA damage, causes cGAS release from the chromatin.

177 Nuclear soluble cGAS is constitutively active.

178 It has been reported that R241E mutation results in the release of cGAS from the chromatin

179 (Volkman, 2019). We stably expressed cGAS R241E mutant in HEK293 cells that are lacking 180 endogenous cGAS. As reported previously (Volkman, 2019), the R241E mutant was only 181 present in the cytosolic and the nuclear soluble fractions (Fig. S3E) and produced a significant 
182 amount of cGAMP without DNA ligand stimulation (Fig. S3F), suggesting the nuclear soluble

183 cGAS is constitutively active.

185 Next, we examined whether the nuclear soluble cGAS induced by HSV-1 was active. We 186 treated RAW 264.7 cells with calf thymus DNA (ctDNA) and HSV-1 followed by subcellular

187 fractionation. As shown in Figure 2E, the cytosolic cGAS had comparable enzymatic activities in 188 cells infected with HSV-1 and treated with ctDNA. However, in vitro cGAS enzymatic assays 189 showed that a significantly higher level of cGAMP was produced by the nuclear soluble extract 190 of the HSV-1- infected RAW 264.7 cells but not the ctDNA-treated cells (Fig. 2E), suggesting

191 the active state of the nuclear soluble cGAS. Furthermore, we compared the cGAS activity in 192 the nuclear soluble fraction in cells infected with different viruses. As shown in Figure 2F, 193 cGAMP production was only detected in the nuclear soluble extract from cells infected with 194 HSV-1, but not other viruses. These data are consistent with the subcellular fraction results that 195 IAV, VSV, and VACV failed to induce nuclear soluble cGAS.

\section{Establish a cell line exclusively expressing nuclear cGAS.}

198 To obtain a cell line exclusively expressing nuclear cGAS, we fused the NLS of SV40 large T-to 199 the C-terminus of cGAS (cGAS-NLS) and the LL/RK mutant (LL/RK-NLS). We then stably 200 transfected these constructs into HEK293 cells. As shown in Figure S4A, although most cGAS201 NLS proteins resided in the nuclear fraction, there was still a small amount of cGAS in the 202 cytosol. However, the LL/RK-NLS was exclusively in the nuclear fractions (Fig. S4A). To further 203 exclude newly synthesized cytosolic cGAS, we generated an inducible system for cGAS, cGAS204 NLS, and the LL/RK-NLS (Fig. 3A) and stably transfected them into HEK293 cells (Fig. S4B). As 205 shown in Figure S4C, cGAS proteins were stable within $8 \mathrm{~h}$ after doxycycline removal. 206 Furthermore, the induced LL/RK-NLS protein exclusively resided in the nucleus by 207 immunofluorescence assays (Fig. S4D). 
209 To determine the functional role of nuclear soluble cGAS, we applied the inducible cGAS

210 expression system into RAW 264.7 knockout macrophages. We generated cGAS knockout in

211 RAW 264.7 macrophages (Fig. S4E). cGAS knockout cells failed to produce IFN $\beta$ and ISGs,

212 such as IP10 and RANTES, when infected with HSV-1 (Fig. S4F). Next, we reconstituted the

213 inducible cGAS and the LL/RK-NLS mutant in cGAS knockout RAW 264.7 macrophages

214 (hereinafter referred to as KO (cGAS) and KO (LL/RK-NLS), respectively) (Fig. S4G).

215 Subcellular fractionation showed that LL/RK-NLS, but not the wild type cGAS, only resided in

216 the chromatin-bound extract of the reconstituted cells after doxycycline induction (Fig. 3B).

218 Nuclear soluble cGAS senses HSV-1 and activates innate immune responses.

219 To examine the role of nuclear cGAS in HSV-1 infection, we infected the cGAS KO (LL/RK-

220 NLS) RAW 264.7 cells with HSV-1. HSV-1 infection induced cGAS release from the chromatin

221 to the nuclear soluble (Fig. 3C), which further corroborates that the nuclear soluble cGAS

222 comes from the chromatin. Next, we compared innate immune responses to HSV-1 d019 in the

223 cGAS KO, KO (cGAS), and KO (LL/RK-NLS) RAW 264.7 cells. As expected, cGAS KO cells

224 failed to respond to HSV-1 infection; however, cGAS and LL/RK-NLS restored the mRNA

225 expression of IFN $\beta$, RANTES, and IP10 (Figs. 3D, S4H-S4I). The reconstitution of cGAS and

226 LL/RK-NLS also rescued TBK1 phosphorylation, the hallmark of activation of IFN production

227 pathways (Fig. 3E). Furthermore, KO (LL/RK-NLS) RAW 264.7 cells produced a significant

228 amount of cGAMP after HSV-1 infection (Fig. 3F), suggesting that nuclear soluble cGAS is 229 activated by HSV-1 infection.

231 It has been reported that cGAS can bind host genomic DNA (Gentili et al., 2019); however, 232 whether nuclear soluble cGAS binds viral DNA is unknown. Thus, we infected the KO (LL/RK- 
233 NLS) cells with the HSV-1 carrying a GFP. After infection, the nuclear soluble extracts were

234 subject to chromatin immunoprecipitation (ChIP) assay. ChIP assays found that cGAS bound 235 GFP and HSV-1 VP16 DNA (Fig. 3G), suggesting that cGAS can sense HSV-1 DNA in the 236 nuclear soluble fraction.

\section{Nuclear cGAS inhibits HSV-1 infection.}

239 We examined whether nuclear soluble cGAS inhibits HSV-1 infection. In this regard, cGAS KO, $240 \mathrm{KO}$ (cGAS), and KO (LL/RK-NLS) cells were infected with HSV-1-GFP. As shown in Figure 4A, 241 wild type cGAS and the LL/RK-NLS mutant rescued the antiviral activity in cGAS knockout cells, 242 evidenced by the reduced number of GFP staining cells. Consistently, the reconstitution of wild 243 type cGAS or the LL/RK-NLS mutant also reduced the expression of viral RNA (Fig. 4B), viral 244 protein (Fig. 4C), and the production of viral particles (Fig. 4D), suggesting that nuclear soluble 245 cGAS limits HSV-1 infection. Furthermore, we examined the role of nuclear cGAS in other 246 viruses. We infected cGAS KO, KO (cGAS), and KO (LL/RK-NLS) cells with HSV-1, VACV, VSV, 247 and IAV reporter viruses. The infection activity of HSV-1, but not other viruses, reduced in the 248 KO (LL/RK-NLS) cells (Fig. 4E), which is consistent with that only HSV-1 induces nuclear 249 soluble cGAS. Interestingly, the reconstitution of wild type cGAS not only restricted HSV-1 250 infection but also impaired the infection of VACV and VSV (Fig. 4E). As VSV and VACV

251 replicate in the cytoplasm, these data suggest that cytosolic cGAS is critical for host defense to 252 cytosolic pathogens. Taken together, our data demonstrate that nuclear soluble cGAS can 253 sense DNA virus infection in the nucleus, instigate innate immune response, and inhibit viral 254 infection. 
257 It has been reported that cGAS locates predominantly in the cytoplasm (Sun et al., 2013),

258 predominantly in the nucleus (Volkman, 2019), on the plasma membrane (Barnett et al., 2019),

259 in both the cytoplasm and the nucleus (Gentili et al., 2019), mitosis-associated nuclear

260 localization (Gentili et al., 2019; Yang et al., 2017), or phosphorylation-mediated nuclear import

261 (Liu et al., 2018). Although the discrepancy is partially due to the cell type, the major reason is

262 that most of the conclusions are based on imaging of the epitope-tagged to cGAS. Most

263 commercial antibodies are not suitable for immunostaining and failed to pass the validation

264 using cGAS knockout human and mouse cells in our hands. A recently developed human cGAS

265 antibody by Cell Signaling Technology passed our in-house validation; therefore, we revisited

266 the subcellular localization of endogenous cGAS by using the validated utility. Consistent with

267 previous studies, we found endogenous cGAS localized in the cytoplasm, nucleus, chromosome,

268 chromatin bridge, and micronuclei. However, different cell lines showed distinct subcellular

269 localization patterns. For example, the localization pattern of cGAS is almost similar in most

270 HFF-1 cells but cGAS could be either cytosolic or nuclear in H1299 cells. Our data imply

271 additional mechanisms might be required to regulate endogenous cGAS localization in different

272 cells.

274 Currently, it is not clear how cGAS enters the nucleus. One study proposed that cGAS nuclear

275 localization results from nuclear envelope breakdown in mitosis or nuclear envelope rupture in

276 interphase (Gentili et al., 2019). Another study reported that the export of nuclear cGAS to the

277 cytosol is required for cytosolic DNA sensing based on the observation of accumulation of

278 cytosolic cGAS after DNA stimulation (Sun et al., 2021). Although the mutation of the NES

279 moderately altered cGAS subcellular localizations, we did not observe a significant

280 accumulation of cytosolic cGAS in multiple cell lines after DNA stimulation. However, our

281 approaches cannot exclude the nucleocytoplasmic shuttling of cGAS. Nonetheless, there is a

282 fair amount of cytosolic cGAS present in the cytosol of unstimulated cells. Another recent study 
283 showed that the collided ribosomes induced nuclear cGAS translocation to the cytosol under

284 translation stress (Wan et al., 2021). The critical question for these models is how cytosolic DNA

285 or translation stress transduces a signal to nuclear cGAS proteins which are chromatin-bound.

286 Logically, chromatin-bound cGAS would be first released into the nuclear soluble fraction, like

287 the R222E and R241E mutants. Then, the nuclear soluble cGAS is exported into the cytosol.

288 However, nuclear soluble cGAS is barely seen during cytosolic DNA stimulation. Whether and

289 how cytosolic DNA stimulation induces cGAS nuclear export needs further investigation in the

290 future.

291

292 It is intriguing to know why cGAS must localize in the nucleus, even it is a sensor that detects 293 cytosolic DNA. One plausible explanation is that the nuclear cGAS senses the viruses or other 294 invading pathogens that can evade the surveillance of cytosolic cGAS by uncoating in the 295 nucleus. Indeed, most DNA viruses, like HSV-1, replicate in the nucleus and expose viral DNA 296 in the nucleus during early infection. Paradoxically, to avoid being constitutively activated in the 297 nucleus, cGAS is tethered to the chromatin. Our study now reveals that HSV-1 infection induces 298 the release of cGAS from the chromatin to the nuclear soluble fraction. The "free" nuclear cGAS 299 is in a DNA-rich environment, and the untethering is sufficient to activate cGAS by either host or 300 viral DNA (Fig. 4F). Taken together, our study demonstrates that nuclear soluble cGAS is critical 301 for sensing viral DNA in the nucleus at the early stage of DNA virus infection. 


\section{MATERIALS AND METHODS}

303 Cells.

304 HEK293 cells (ATCC, \# CRL-1573), RAW 264.7 (ATCC, \# TIB-71), NCI-H596 cells (ATCC, 305 HTB-178), HFF-1 (ATCC, \# SCRC-1041), MDA-MB-231 (Sigma, 92020424-1VL), and Vero 306 cells (ATCC, \# CCL-81) were maintained in Dulbecco's Modified Eagle Medium (Life 307 Technologies, \# 11995-065) containing antibiotics (Life Technologies, \# 15140-122) and 10\% 308 fetal bovine serum (Life Technologies, \# 26140-079). NCl-H1299 cells (ATCC, \# CRL-5083), 309 THP-1 cells (ATCC) were cultured in RPMI Medium 1640 (Life Technologies, \# 11875-093) plus

$31010 \%$ fetal bovine serum. A549 cells (ATCC, \# CCL-185) were cultured in RPMI Medium 1640 311 (Life Technologies, \# 11875-093) plus 10\% fetal bovine serum and $1 \times$ MEM Non-Essential 312 Amino Acids Solution (Life Technologies, \# 11140-050).

Viruses.

315 HSV-1 KOS strain was purchased from ATCC (\#VR-1493). Viral titration was performed as the 316 following. Vero cells were infected with a serial diluted HSV-1. After $1 \square \mathrm{h}$, the medium was 317 removed and replaced by the DMEM plus 5\% FBS and $1 \%$ agarose. Cells were examined for 318 cytopathic effects to determine TCID50 or were fixed using the methanol-acetic acid (3:1) 319 fixative and stained using a Coomassie blue solution to determine $\mathrm{MOI}$.

321 Plasmids.

322 Mouse and human cGAS cDNA were synthesized and cloned into pCMV-3Tag-8 to generate 323 mcGAS-FLAG and hcGAS-FLAG. Point mutations and deletions of cGAS were constructed 324 using a Q5® Site-Directed Mutagenesis Kit (New England Biolabs, \# E0554S). 
327 Primary antibodies: Anti-ß-actin (Abcam, \# ab8227), anti-FLAG (Sigma, \# F3165), anti-TBK1

328 (Cell Signaling Technology, \# 3504S], anti-phospho-TBK1 (Ser172) (Cell Signaling Technology, 329 \# 5483S), anti-C1QBP (Cell Signaling Technology, \# 6502S), anti-a-Tubulin (Cell Signaling 330 Technology, \# 3873S), anti-Histone H3 (Cell Signaling Technology, \# 4499S), anti-mouse 331 STING (Cell Signaling Technology, \# 50494S), anti-human STING (R\&D Systems, \# MAB7169332 SP), anti-vimentin (R\&D Systems, \# MAB21052-SP), anti-ICP8 (Abcam, \# ab20194), anti-NP

333 (GenScript, \# A01506-40), anti-y-H2AX (ABclonal, \# AP0099), anti-human cGAS (Cell Signaling 334 Technology, \#79978), anti-mouse cGAS (Cell Signaling Technology, \#31659).

336 Secondary antibodies: Goat anti-Mouse IgG-HRP [Bethyl Laboratories, \# A90-116P, WB 337 (1:10,000)], Goat anti-Rabbit IgG-HRP [Bethyl Laboratories, \# A120-201P, WB (1:10,000)], 338 Alexa Fluor 594 Goat Anti-Mouse lgG $(H+L)$ [Life Technologies, \# A11005, IFA (1:200)], Alexa 339 Fluor 488 Goat Anti-Rabbit IgG (H+L) [Life Technologies, \# A11034, IFA (1:200)].

341 Subcellular fractionation

342 Approximately $2 \times 10^{6}$ cells were fractionated using the Subcellular Protein Fractionation Kit for 343 Cultured Cells (Thermo scientific, \#78840).

\section{CHIP-qPCR assay}

346 Briefly, HEK293 cells stably expressing FLAG-tagged LL/RK-NLS were infected with HSV-1347 GFP virus for $16 \mathrm{~h}$. Cells were cross-linked with formaldehyde for $10 \mathrm{~min}$, followed by adding $348125 \mathrm{mM}$ glycine to stop the cross-linking. Cells were then washed with $1 \mathrm{ml} 1 \times$ PBS two times 349 and centrifuged for $5 \mathrm{~min}$ at $4^{\circ} \mathrm{C}, 1,000 \times \mathrm{g}$. The pelleted cells were used for subcellular 350 fractionation. The nuclear soluble extracts were immunoprecipitated with the anti-FLAG beads. 
351 After three times washings with 1 x PBS, cGAS complexes were eluted using FLAG peptides,

352 and then the elutes were subject to qPCR assay.

354 cGAMP assay

355 Cells were collected by centrifuging at $500 \mathrm{xg}$ for 5 minutes, and then resuspended and lysed in

356 PBS or the Immunoassay Buffer C (Cayman chemical, \# 401703) through boiling at $95^{\circ} \mathrm{C}$ for 10

357 minutes. The lysates were then centrifuged for $30 \mathrm{~min}$ at $4{ }^{\circ} \mathrm{C}, 10,000 \times \mathrm{g}$. Supernatants were

358 collected for cGAMP ELISA assays. For subcellular fractions, the samples were diluted $10 \mathrm{x}$

359 with the Immunoassay Buffer C (Cayman chemical, \# 401703). The cGAMP amount was

360 determined by ELISA assays according to the manufacture's protocols (Cayman chemical, \# $361501700)$.

\section{Sample preparation, Western blotting, and immunoprecipitation}

364 Approximately $1 \times 10^{6}$ cells were lysed in $500 \mu$ of tandem affinity purification (TAP) lysis buffer $365\left[50 \mathrm{mM}\right.$ Tris- $\mathrm{HCl}(\mathrm{pH} 7.5), 10 \mathrm{mM} \mathrm{MgCl}_{2}, 100 \mathrm{mM} \mathrm{NaCl}, 0.5 \%$ Nonidet P40, 10\% glycerol, the 366 Complete EDTA-free protease inhibitor cocktail tablets (Roche, \# 11873580001)] for 30 min at 4

$367 \square{ }^{\circ} \mathrm{C}$. The lysates were then centrifuged for $30 \mathrm{~min}$ at $15,000 \mathrm{rpm}$. Supernatants were mixed 368 with the Lane Marker Reducing Sample Buffer (Thermo Fisher Scientific, \# 39000) and boiled at $36995^{\circ} \mathrm{C}$ for 5 minutes.

371 Western blotting and immunoprecipitation were performed as described in a previous study

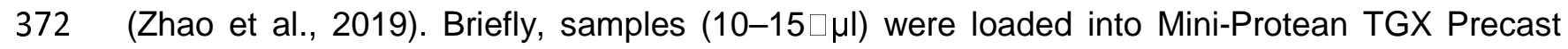
373 Gels, 15 well (Bio-Rad, \# 456-103), and run in $1 \times$ Tris/Glycine/SDS Buffer (Bio-Rad, \# 161$3740732)$ for $60 \square \min$ at $140 \square$ V. Protein samples were transferred to Immun-Blot PVDF Membranes 375 (Bio-Rad, \# 162-0177) in $1 \times$ Tris/Glycine buffer (Bio-Rad, \# 161-0734) at 70 $\square \mathrm{V}$ for 60 $\square \mathrm{min}$. 
376 PVDF membranes were blocked in $1 \times$ TBS buffer (Bio-Rad, \# 170-6435) containing 5\%

377 Blotting-Grade Blocker (Bio-Rad, \# 170-6404) for 1 $\square$ h. After washing with $1 \times$ TBS buffer for a 378 total of $30 \square \min (10 \mathrm{~min}$ each time, repeat 3 times), the membrane blot was incubated with the 379 appropriately diluted primary antibody in antibody dilution buffer $(1 \times$ TBS, 5\% BSA, $0.02 \%$ 380 sodium azide) at $4 \square^{\circ} \mathrm{C}$ for $16 \square \mathrm{h}$. Then, the blot was washed three times with $1 \times$ TBS (each 381 time for $10 \square \mathrm{min}$ ) and incubated with secondary HRP-conjugated antibody in antibody dilution

382 buffer (1:10,000 dilution) at room temperature for $1 \square \mathrm{h}$. After three washes with $1 \times$ TBS (each 383 time for $10 \square \min$ ), the blot was incubated with Clarity Western ECL Substrate (Bio-Rad, \# 170$3845060)$ for $1-2 \square \min$. The membrane was removed from the substrates and then exposed to the 385 Amersham imager 600 (GE Healthcare Life Sciences, Marlborough, MA).

\section{Immunofluorescence assay.}

388 Cells were cultured in the Lab-Tek II CC2 Chamber Slide System 4-well (Thermo Fisher 389 Scientific, \# 154917). After the indicated treatment, the cells were fixed and permeabilized in 390 cold methanol for $10 \square \min$ at $-20 \square^{\circ} \mathrm{C}$. Then, the slides were washed with $1 \times$ PBS for $10 \square$ min 391 and blocked with Odyssey Blocking Buffer (LI-COR Biosciences, \# 927-40000) for 1 $\square$ h. The 392 slides were incubated in Odyssey Blocking Buffer with appropriately diluted primary antibodies 393 at $4 \square{ }^{\circ} \mathrm{C}$ for $16 \square \mathrm{h}$. After 3 washes (10 $\square$ min per wash) with $1 \times$ PBS, the cells were incubated 394 with the corresponding Alexa Fluor conjugated secondary antibodies (Life Technologies) for $3951 \square \mathrm{h}$ at room temperature. The slides were washed three times $(10 \square$ min each time) with $1 \times$ 396 PBS and counterstained with $300 \square \mathrm{nM}$ DAPI for $1 \square \mathrm{min}$, followed by washing with $1 \times$ PBS for $3971 \square \min$. After air-drying, the slides were sealed with Gold Seal Cover Glass (Electron 398 Microscopy Sciences, \# 3223) using Fluoro-gel (Electron Microscopy Sciences, \# 17985-10). 
401 Total RNA was prepared using the RNeasy Mini Kit (Qiagen, \# 74106). Five hundred

402 nanograms of RNA was reverse transcribed into cDNA using the QuantiTect reverse

403 transcription kit (Qiagen, \# 205311). For one real-time reaction, $10 \mu \mathrm{l}$ of SYBR Green PCR

404 reaction mix (Eurogentec), including $100 \mathrm{ng}$ of the synthesized cDNA plus an appropriate

405 oligonucleotide primer pair, were analyzed on a 7500 Fast Real-time PCR System (Applied

406 Biosystems). The comparative $\mathrm{C} t$ method was used to determine the relative mRNA expression

407 of genes normalized by the housekeeping gene GAPDH. The primer sequences: mouse Gapdh,

408 forward primer 5`- GCGGCACGTCAGATCCA $\quad 3{ }^{`}$, reverse primer 5`-

409 CATGGCCTTCCGTGTTCCTA -3`; mouse Ifnb1, forward primer $5^{`}$ -

410 CAGCTCCAAGAAAGGACGAAC -3`, reverse primer 5`- GGCAGTGTAACTCTTCTGCAT -3`;

411 mouse CxCl10 (IP10), forward primer 5`- CCAAGTGCTGCCGTCATTTTC -3`, reverse primer 5`-

412 GGCTCGCAGGGATGATTTCAA -3`; mouse Ccl5 (RANTES), forward primer 5`-

413 GCTGCTTTGCCTACCTCTCC -3`, reverse primer 5`-TCGAGTGACAAACACGACTGC-3`;

414 GFP, forward primer 5`- AAGGGCATCGACTTCAAGG -3`, reverse primer 5`-

415 TGCTTGTCGGCCATGATATAG $-3^{`}$; HSV-1 VP16, forward primer $5^{`}$ -

416 GGACTGTATTCCAGCTTCAC -3`, reverse primer 5` CGTCCTCGCCGTCTAAGTG -3`.

\section{Plasmid transfection.}

419 HEK293 and RAW cells were transfected using Lipofectamine 3000 or Lipofectamine LTX 420 Transfection Reagent (Life Technologies, \# L3000015) according to the manufacturer's

421 protocol.

\section{CRISPR/Cas9.}

423 The single guide RNA (sgRNA) targeting sequences: mouse cGAS sgRNA1: forward primer $5{ }^{`}-$

424 CACCGACGCAAAGATATCTCGGAGG $-3^{`}$, reverse primer $\quad 5^{`}$ AAAC 425 CCTCCGAGATATCTTTGCGTC-3`; sgRNA2 forward primer 5 ${ }^{`}$-CACCG 
427 3`. SgRNA3 forward primer 5`- CACCGGCGGACGGCTTCTTAGCGCG -3`; reverse primer 5`-

428 AAACCGCGCTAAGAAGCCGTCCGCC -3`. The sgRNA was cloned into lentiCRISPR v2 vector

429 (Sanjana et al., 2014) (Addgene). The lentiviral construct was transfected with psPAX2 and 430 PMD2G into HEK293T cells using PEI. After $72 \mathrm{~h}$, the media containing lentivirus were 431 collected. The targeted cells were infected with the media containing the lentivirus 432 supplemented with $10 \mu \mathrm{g} / \mathrm{ml}$ polybrene. Cells were selected with $10 \mu \mathrm{g} / \mathrm{ml}$ puromycin for 14 433 days. Single clones were expanded for knockout confirmation by Western blotting.

\section{Stable Cell Line Selection}

436 HEK293 cells and RAW 264.7 cells were transfected with the relative constructs or infected with

437 the media containing the lentivirus supplemented with $10 \mu \mathrm{g} / \mathrm{ml}$ polybrene. Cells were selected 438 with $200 \mu \mathrm{g} / \mathrm{ml}$ hygromycin or $10 \mu \mathrm{g} / \mathrm{ml}$ puromycin for 14 days. Stable cell lines were validated 439 by Western blotting.

441 Statistics and Reproducibility.

442 The sample size was sufficient for data analyses. Data were statistically analyzed using the

443 software GraphPad Prism 9. Significant differences between the indicated pairs of conditions 444 are shown by asterisks $\left({ }^{*} P<0.05\right.$; ${ }^{* *} P<0.01$; ${ }^{* * *} P<0.001$; $\left.{ }^{* * *} P<0.0001\right)$.

\section{Author Contributions}

447 SL conceived and supervised the project. SL, YW, KS, WH, and LW designed the study. YW, $448 \mathrm{KS}, \mathrm{WH}$, and LW performed the experiments. SL, YW, KS, WH, and LW analyzed the data. All 449 authors contributed to manuscript writing, revision, read, and approved the submitted version. 


\section{ACKNOWLEDGEMENT}

452 This research was funded by the National Institutes of Health (R21Al137750 and R01Al141399

453 to S.L.).

454

455 Competing interests

456 The authors declare no competing interests. 


\section{REFERENCES}

Barber, G.N. (2015). STING: infection, inflammation and cancer. Nat Rev Immunol 15, 760-770. Barnett, K.C., Coronas-Serna, J.M., Zhou, W., Ernandes, M.J., Cao, A., Kranzusch, P.J., and Kagan, J.C. (2019). Phosphoinositide Interactions Position cGAS at the Plasma Membrane to Ensure Efficient Distinction between Self- and Viral DNA. Cell 176, 1432-1446 e1411. Boyer, J.A., Spangler, C.J., Strauss, J.D., Cesmat, A.P., Liu, P., McGinty, R.K., and Zhang, Q. (2020). Structural basis of nucleosome-dependent cGAS inhibition. Science. Cai, X., Chiu, Y.H., and Chen, Z.J. (2014). The cGAS-cGAMP-STING pathway of cytosolic DNA sensing and signaling. Mol Cell 54, 289-296. Cao, D., Han, X., Fan, X., Xu, R.M., and Zhang, X. (2020). Structural basis for nucleosomemediated inhibition of cGAS activity. Cell Res 30, 1088-1097. Chen, Q., Sun, L., and Chen, Z.J. (2016). Regulation and function of the cGAS-STING pathway of cytosolic DNA sensing. Nat Immunol 17, 1142-1149.

472 Eidson, K.M., Hobbs, W.E., Manning, B.J., Carlson, P., and DeLuca, N.A. (2002). Expression of herpes simplex virus ICPO inhibits the induction of interferon-stimulated genes by viral infection. J Virol 76, 2180-2191. Fitzgerald, K.A., McWhirter, S.M., Faia, K.L., Rowe, D.C., Latz, E., Golenbock, D.T., Coyle, A.J., Liao, S.M., and Maniatis, T. (2003). IKKepsilon and TBK1 are essential components of the IRF3 signaling pathway. Nat Immunol 4, 491-496. Gentili, M., Lahaye, X., Nadalin, F., Nader, G.P.F., Lombardi, E.P., Herve, S., De Silva, N.S., Rookhuizen, D.C., Zueva, E., Goudot, C., et al. (2019). The N-Terminal Domain of cGAS Determines Preferential Association with Centromeric DNA and Innate Immune Activation in the Nucleus. Cell Rep 26, 3798. Hemmi, H., Takeuchi, O., Sato, S., Yamamoto, M., Kaisho, T., Sanjo, H., Kawai, T., Hoshino, K., lipopolysaccharide and double stranded RNA signaling and viral infection. J Exp Med 199, 16411650. unifying concepts in sensing and responding to cytosolic nucleic acids. Nat Rev Immunol 14, 521-528.

Hu, J., Lieb, J.D., Sancar, A., and Adar, S. (2016). Cisplatin DNA damage and repair maps of the human genome at single-nucleotide resolution. Proc Natl Acad Sci U S A 113, 11507-11512. Ishikawa, H., and Barber, G.N. (2008). STING is an endoplasmic reticulum adaptor that facilitates innate immune signalling. Nature 455, 674-678.

Kujirai, T., Zierhut, C., Takizawa, Y., Kim, R., Negishi, L., Uruma, N., Hirai, S., Funabiki, H., and Kurumizaka, H. (2020). Structural basis for the inhibition of cGAS by nucleosomes. Science. Li, T., Huang, T., Du, M., Chen, X., Du, F., Ren, J., and Chen, Z.J. (2021). Phosphorylation and chromatin tethering prevent CGAS activation during mitosis. Science 371.

Liu, H., Zhang, H., Wu, X., Ma, D., Wu, J., Wang, L., Jiang, Y., Fei, Y., Zhu, C., Tan, R., et al. (2018). Nuclear cGAS suppresses DNA repair and promotes tumorigenesis. Nature 563, 131-136. Mackenzie, K.J., Carroll, P., Martin, C.A., Murina, O., Fluteau, A., Simpson, D.J., Olova, N., Sutcliffe, H., Rainger, J.K., Leitch, A., et al. (2017). cGAS surveillance of micronuclei links genome instability to innate immunity. Nature $548,461-465$. 
501 McWhirter, S.M., Fitzgerald, K.A., Rosains, J., Rowe, D.C., Golenbock, D.T., and Maniatis, T.

502 (2004). IFN-regulatory factor 3-dependent gene expression is defective in Tbk1-deficient mouse 503 embryonic fibroblasts. Proc Natl Acad Sci U S A 101, 233-238.

504 Michalski, S., de Oliveira Mann, C.C., Stafford, C., Witte, G., Bartho, J., Lammens, K., Hornung, V., and Hopfner, K.P. (2020). Structural basis for sequestration and autoinhibition of cGAS by chromatin. Nature. Kozicka, Z., Guey, B., et al. (2020). Structural mechanism of cGAS inhibition by the nucleosome. Nature. Pokatayev, V., Hasin, N., Chon, H., Cerritelli, S.M., Sakhuja, K., Ward, J.M., Morris, H.D., Yan, N., and Crouch, R.J. (2016). RNase H2 catalytic core Aicardi-Goutieres syndrome-related mutant invokes cGAS-STING innate immune-sensing pathway in mice. J Exp Med 213, 329-336. Sanjana, N.E., Shalem, O., and Zhang, F. (2014). Improved vectors and genome-wide libraries for CRISPR screening. Nat Methods 11, 783-784. Sharma, S., tenOever, B.R., Grandvaux, N., Zhou, G.P., Lin, R., and Hiscott, J. (2003). Triggering the interferon antiviral response through an IKK-related pathway. Science 300, 1148-1151. Sun, H., Huang, Y., Mei, S., Xu, F., Liu, X., Zhao, F., Yin, L., Zhang, D., Wei, L., Wu, C., et al. (2021). A Nuclear Export Signal Is Required for CGAS to Sense Cytosolic DNA. Cell Rep 34, 108586. Sun, L., Wu, J., Du, F., Chen, X., and Chen, Z.J. (2013). Cyclic GMP-AMP synthase is a cytosolic DNA sensor that activates the type I interferon pathway. Science 339, 786-791. (2009). ERIS, an endoplasmic reticulum IFN stimulator, activates innate immune signaling through dimerization. Proc Natl Acad Sci U S A 106, 8653-8658. DNA signaling pathway. Sci Signal 5, ra20.

526 Volkman, H.E., Cambier, S., Gray, E. E. \& Stetson, D. B. (2019). Tight nuclear tethering of cGAS is essential for preventing autoreactivity. Elife. Wan, L., Juszkiewicz, S., Blears, D., Bajpe, P.K., Han, Z., Faull, P., Mitter, R., Stewart, A., Snijders, A.P., Hegde, R.S., and Svejstrup, J.Q. (2021). Translation stress and collided ribosomes are coactivators of cGAS. Mol Cell. Yang, H., Wang, H., Ren, J., Chen, Q., and Chen, Z.J. (2017). cGAS is essential for cellular senescence. Proc Natl Acad Sci U S A 114, E4612-E4620. Sensing Pathways. Trends Immunol 38, 194-205. containing mixed phosphodiester linkages is an endogenous high-affinity ligand for STING. Molecular cell 51, 226-235. The Molecular Basis of Tight Nuclear Tethering and Inactivation of cGAS. Nature. al. (2019). Non-proteolytic ubiquitination of OTULIN regulates NF-kappaB signaling pathway. J Mol Cell Biol. 
543 Zhong, B., Yang, Y., Li, S., Wang, Y.Y., Li, Y., Diao, F., Lei, C., He, X., Zhang, L., Tien, P., and Shu, 544 H.B. (2008). The adaptor protein MITA links virus-sensing receptors to IRF3 transcription factor 545 activation. Immunity 29, 538-550.

546 Zhong, L., Hu, M.M., Bian, L.J., Liu, Y., Chen, Q., and Shu, H.B. (2020). Phosphorylation of cGAS 547 by CDK1 impairs self-DNA sensing in mitosis. Cell Discov 6, 26. 
551 Figure. 1 The N-terminal domain and NES regulate cGAS nuclear localization. (A) The cell

552 lysates of RAW 264.7 macrophages were separated by 15-55\% sucrose density centrifugation.

553 Fractions were blotted as indicated. The fraction of thyroglobulin (660 kDa), a protein standard,

554 was indicated. (B) $\mathrm{H} 1299$ cells were stimulated with or without $1 \mu \mathrm{g} / \mathrm{ml}$ calf thymus DNA

555 (ctDNA) for $4 \square \mathrm{h}$. Then, the cell lysates were fractionated into five fractions: cytoplasmic,

556 membrane, nuclear soluble, chromatin-bound, and cytoskeletal. The fractions were blotted as

557 indicated. STING: membrane marker; TBK1 and $\beta$-actin: cytosolic marker; H3: nuclear marker;

558 vimentin: cytoskeletal marker. (C) Wild type (WT) and cGAS knockout (KO) H1299 cells were

559 either mock stimulated or transfected with ctDNA. After $4 \mathrm{~h}$, cells were fixed and stained as

560 indicated. cGAS: green; DAPI, blue. Bar $=10 \mu \mathrm{m}$. (D) Representative cGAS localization in

561 unstimulated H1299 WT cells in (C). (i) nucleoli; (ii) micronucleus; (iii) chromosome; (iv)

562 chromatin bridge; (v) perinuclear region. Arrows indicate each distinct localization in (i) to (v).

563 cGAS: green; DAPI, blue. Bar $=10 \mu \mathrm{m}$. (E) Schematic of the LL/RK mutation in the nuclear

564 export signal of cGAS. Red arrows indicate the mutated sites, and the green frame indicates the

565 introduction of a nuclear import signal (NLS) caused by the mutations. (F) Immunofluorescence

566 assays of HEK293 cells stably expressing FLAG-tagged mouse cGAS (mcGAS) or the indicated

567 LL/RK mutant. FLAG: red; DAPI, blue. Bar $=10 \mu \mathrm{m}$. The summary of the subcellular

568 localization of cGAS and LL/RK mutant was shown in the left panel. C: cytosolic; N: nuclear;

$569 \mathrm{C}+\mathrm{N}$ : cytosolic and nuclear. Data represent means \pm s.d. of three independent experiments (>

570200 cells were counted in each field and five fields were counted per experiment). (G) HEK293

571 cells stably expressing the cGAS delN with LL/RK mutation (deIN-LL/RK) were stimulated with

572 or without $1 \mu \mathrm{g} / \mathrm{ml}$ ctDNA for $4 \square \mathrm{h}$. Then, the cell lysates were fractionated into five fractions:

573 cytoplasmic, membrane, nuclear soluble, chromatin-bound, and cytoskeletal. The fractions were 
574 blotted as indicated. C1QBP: membrane marker; TBK1 and $\alpha$-tubilin: cytosolic marker; H3:

575 nuclear marker.

576

577 Figure. 2. HSV-1 infection causes cGAS release from the chromatin to the nuclear soluble

578 fraction. (A) RAW 264.7 macrophages were infected with HSV-1 for $16 \mathrm{~h}$, and then the

579 subcellular fractions of cell lysates were blotted as indicated. The arrow indicates nuclear

580 soluble cGAS after viral infection. (B) RAW 264.7 cells were mock-infected or infected with 0.1

$581 \mathrm{MOI}$ of HSV-1 d109 for $16 \mathrm{~h}$. Cell lysates were fractionated and blotted as indicated. The arrow

582 indicates nuclear soluble cGAS after viral infection. (C) RAW 264.7 cells were pretreated

583 without or with $8 \mu \mathrm{g} / \mathrm{ml}$ of acyclovir for $16 \mathrm{~h}$, followed by infection with $0.1 \mathrm{MOI}$ of HSV-1 for $16 \mathrm{~h}$.

584 Cell lysates were fractionated and blotted as indicated. The arrow indicates nuclear soluble

585 cGAS. (D) RAW 264.7 macrophages were treated with dimethylformamide (DMF) or $50 \mu \mathrm{M}$

586 cisplatin for $3 \mathrm{~h}$. Cell lysates were fractionated and blotted as indicated. STING: membrane

587 marker; $\alpha$-tubulin: cytosolic marker; H3: nuclear marker; ICP8: HSV-1 viral protein; $\gamma$-H2A.X:

588 DNA damage marker. (E) RAW 264.7 cells were transfected with ctDNA or infected with HSV-1

589 for $8 \mathrm{~h}$. The cytoplasmic, nuclear soluble and chromatin-bound fractions were harvested for in

590 vitro cGAS enzymatic activity assays. (F) RAW 264.7 cells were infected with HSV-1, VACV,

591 IAV, or VSV for $8 \mathrm{~h}$. The nuclear soluble extracts were harvested for in vitro cGAS enzymatic

592 activity assays.

594 Figure. 3. Nuclear soluble cGAS senses HSV-1 and instigates innate immune response.

595 (A) Schematic of the doxycycline (Dox)-induced cGAS-NLS and LL/RK-NLS constructs. TRE:

596 Tet Response Element; Puro: puromycin; T2A: Thosea asigna virus 2A-like peptide. (B) cGAS

597 RAW 264.7 knockout macrophages reconstituted with the inducible cGAS or the inducible

598 LL/RK-NLS mutant were fractionated into five fractions and blotted as indicated. STING: 
599 membrane marker; $\alpha$-tubulin: cytosolic marker; ICP8: HSV-1 viral protein; H3: nuclear marker.

600 (C) The cGAS KO (LL/RK-NLS) RAW 264.7 cells were mock-infected or infected with HSV-1 for

60116 h. Then, cells were fractionated into five fractions and blotted as indicated. STING:

602 membrane marker; TBK1 and $\alpha$-tubulin: cytosolic marker; H3: nuclear marker. Red arrow

603 indicates nuclear soluble cGAS. (D) The cGAS KO, KO (cGAS), and KO (LL/RK-NLS) RAW

604264.7 cells were infected with $1 \mathrm{MOI}$ of HSV-1 d109 for designated hours post-infection (h.p.i.).

605 Real-time PCR was performed to determine the relative IFN $\beta$ mRNA levels. Data represent

606 means \pm s.d. of three independent experiments. All experiments were repeated three times. The

$607 P$-value was calculated by two-way ANOVA followed by Dunnett's multiple comparisons test ( $P$

$608<0.05,{ }^{* * *} P<0.001$ ). (E) The cGAS KO, KO (cGAS), and KO (LL/RK-NLS) RAW 264.7

609 macrophages were infected with $1 \mathrm{MOI}$ of HSV-1 for indicated times. Cell lysates were collected

610 and blotted as indicated. Band densitometry was calculated by Image J. The relative ratio of

611 phosphorylated TBK1 to total TBK1 in each lane was indicated. (F) cGAS KO, KO (cGAS), and

612 KO (LL/RK-NLS) RAW 264.7 macrophages were infected with $1 \mathrm{MOI}$ of HSV-1 for $8 \mathrm{~h}$. The

613 amount of cGAMP in each cell line was determined by ELISA assays. All experiments were

614 repeated three times. The $P$ value was calculated by one-way ANOVA followed by Tukey's

615 multiple comparisons test $\left({ }^{*} P<0.05\right.$, $\left.{ }^{* *} P<0.001\right)$. (G) HEK293 cells stably expressing FLAG

616 tagged LL/RK-NLS mutant were infected with HSV-1-GFP for $8 \mathrm{~h}$. ChIP assays were performed

617 using the anti-FLAG antibody. Real-time PCR was performed to determine the relative binding

618 amount of GFP and viral gene VP16. Data represent means \pm s.d. of three independent

619 experiments. All experiments were repeated three times. The $P$-value was calculated by two-

620 way ANOVA followed by Dunnett's multiple comparisons test ( $\left.{ }^{* *} P<0.001,{ }^{* * * *} P<0.0001\right)$.

621

622 Figure. 4. Nuclear soluble cGAS inhibits HSV-1 infection. (A) cGAS KO, KO (cGAS), KO

623 (LL/RK-NLS) RAW 264.7 cells were infected with $0.1 \mathrm{MOI}$ of HSV-1-GFP for 16 $\square$ h. Cells 
624 expressing GFP were examined and counted under a fluorescence microscope. The relative

625 infection was determined by the ratio of positive cells. Data represent means \pm s.d. of three

626 independent experiments (> 200 cells were counted in each field and five fields were counted

627 per experiment). The $P$-value was calculated by one-way ANOVA followed by Dunnett's multiple

628 comparisons test $\left.{ }^{* * *} P<0.0001\right)$. (B) The cGAS KO, KO (cGAS), KO (LL/RK-NLS) RAW

629264.7 cells were infected with $1 \mathrm{MOI}$ of HSV-1 for $16 \square \mathrm{h}$. Then, cells were collected for RNA

630 extraction. The RNA levels of HSV-1 VP16 were determined by real-time PCR. All experiments

631 were repeated three times. The $P$-value was calculated by two-way ANOVA followed by

632 Dunnett's multiple comparisons test $\left.{ }^{* * *} P<0.0001\right)$. (C) The cGAS KO, KO (cGAS), KO

633 (LL/RK-NLS) RAW 264.7 macrophages were infected with $1 \mathrm{MOI}$ of HSV-1 for indicated times.

634 Cell lysates were collected and blotted as indicated. ICP8 is an HSV-1 viral protein. (D) The

635 cGAS KO, KO (cGAS), KO (LL/RK-NLS) RAW 264.7 macrophages were infected with $0.01 \mathrm{MOI}$

636 of HSV-1 for the indicated days. HSV-1 titers were determined in Vero cells. Data represent

637 means \pm s.d. of three independent experiments. All experiments were repeated three times. The

$638 P$-value was calculated by two-way ANOVA followed by Dunnett's multiple comparisons test $\left({ }^{*} P\right.$

$639<0.05$ vs. the cGAS KO cells). (E) The cGAS KO, KO (cGAS), KO (LL/RK-NLS) RAW 264.7

640 macrophages were infected with HSV-1-Luc, VACV-Luc, VSV-Luc, or IAV-Gluc for 16 h.

641 Luciferase activities were measured to determine the relative infection activity. Data represent

642 means \pm s.d. of three independent experiments. All experiments were repeated three times. The

$643 P$-value was calculated by two-way ANOVA followed by Dunnett's multiple comparisons test $\left({ }^{*} P\right.$

$644<0.05,{ }^{* \star * *} P<0.0001$, ns: not significant). (F) Model of nuclear soluble cGAS sensing DNA

645 virus infection.

646 
bioRxiv preprint doi: https://doi.org/10.1101/2021.08.27.457948; this version posted August 28, 2021. The copyright holder for this preprint

A (which was not certified by peer review) is the author/funder. All rights reserved. No reuse allowed without permission.

$B^{\circ}$
$15 \%$
Fraction \#

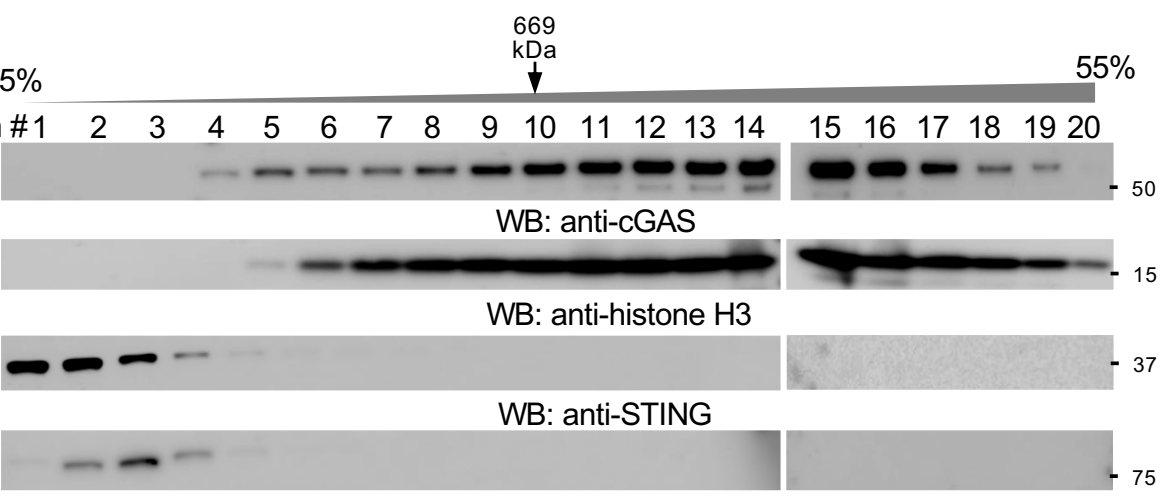

WB: anti-TBK1

C
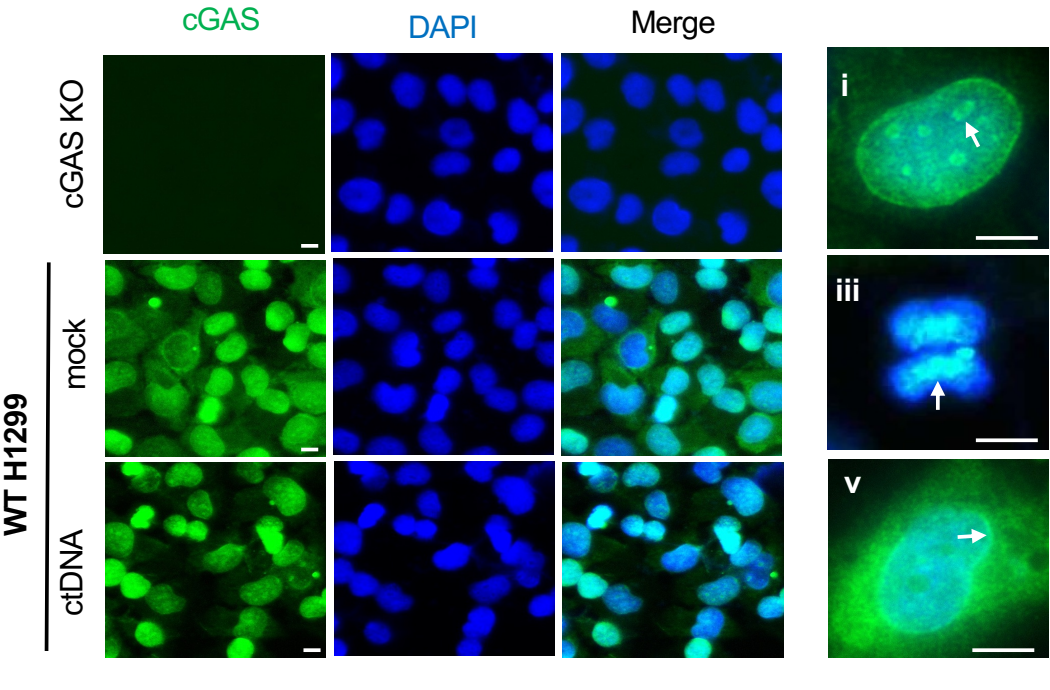

E

F

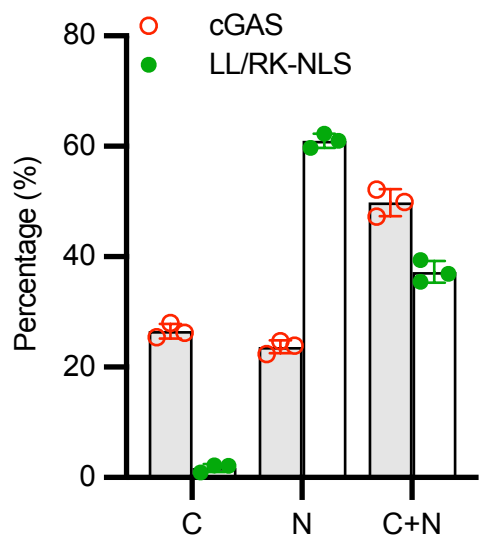

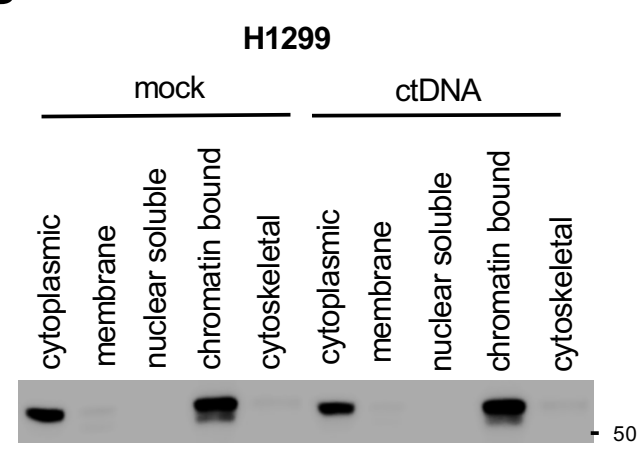

WB: anti-cGAS

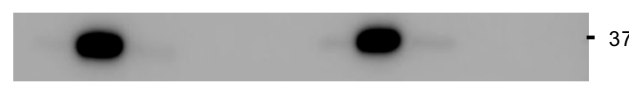

WB: anti-STING
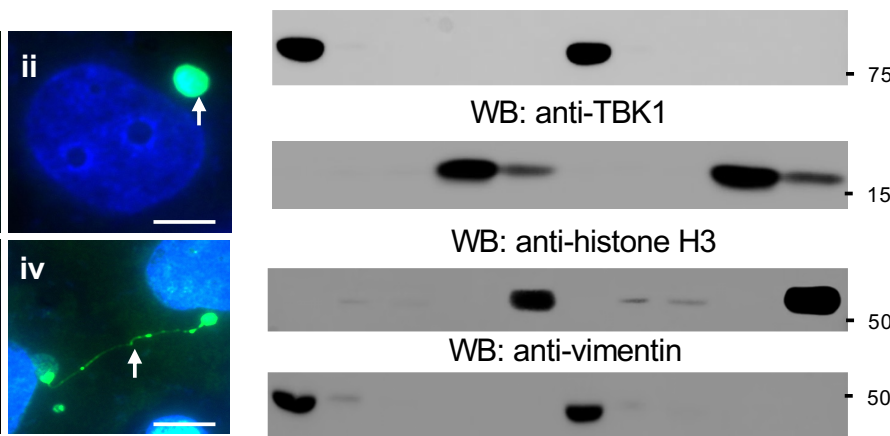

WB: anti-histone $\mathrm{H} 3$

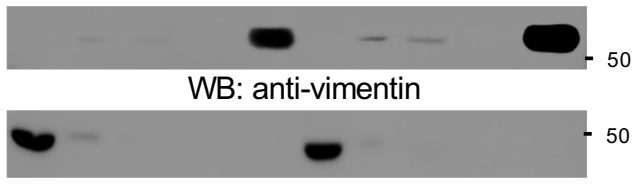

WB: anti- $\beta$-actin

HEK293

delN-LL/RK

$G$

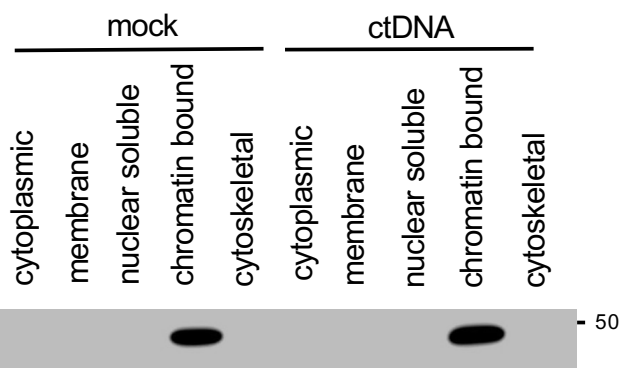

WB: anti-cGAS

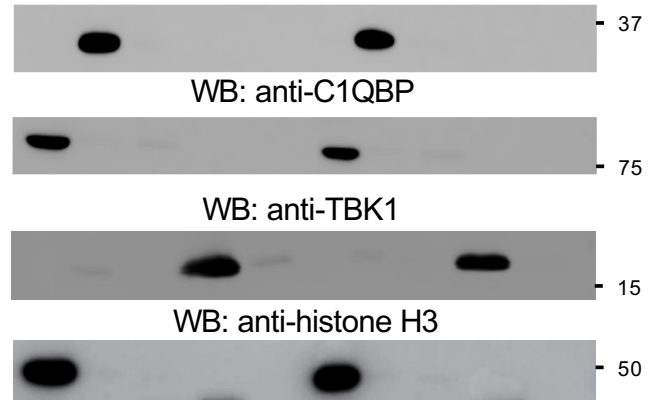

WB: anti- $\alpha$-tubulin 
A
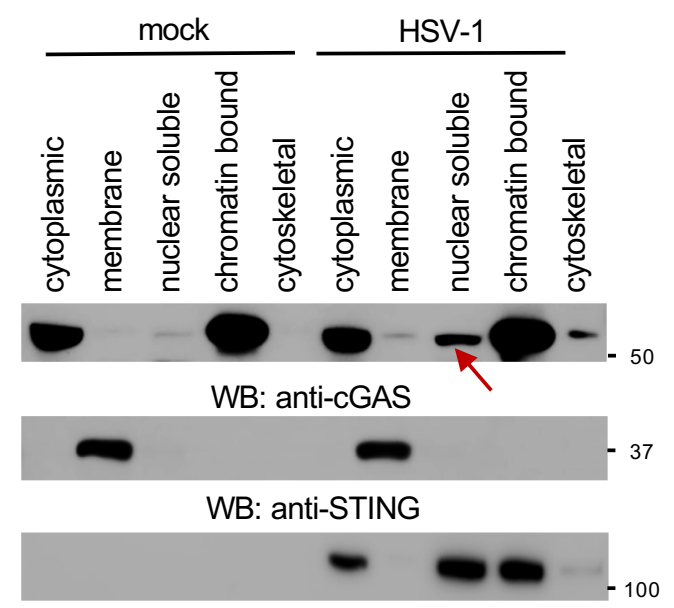

WB: anti-ICP8

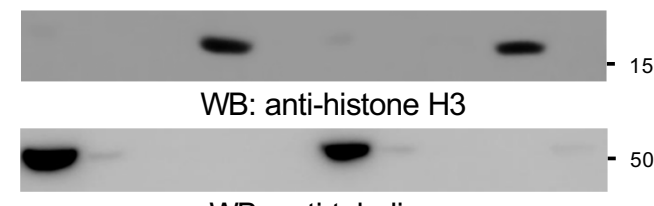

WB: anti-tubulin

D

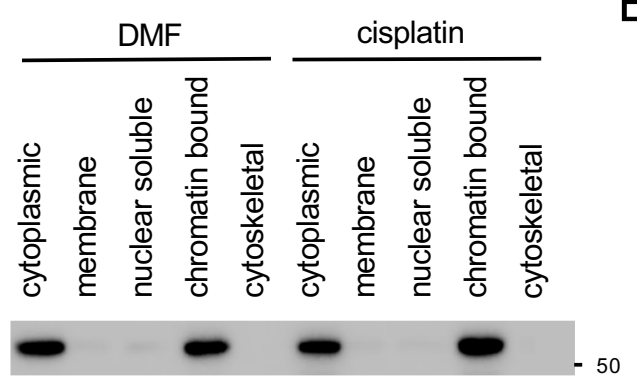

WB: anti-cGAS

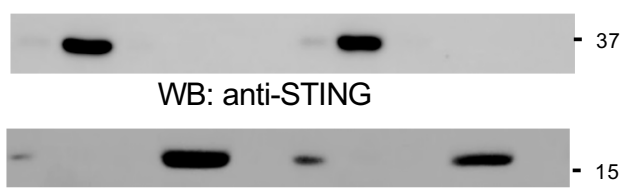

WB: anti-histone $\mathrm{H} 3$

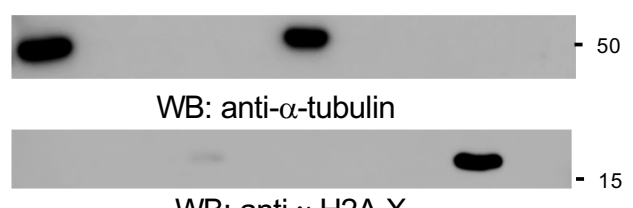

WB: anti- $\gamma-\mathrm{H} 2 \mathrm{~A} . \mathrm{X}$
B

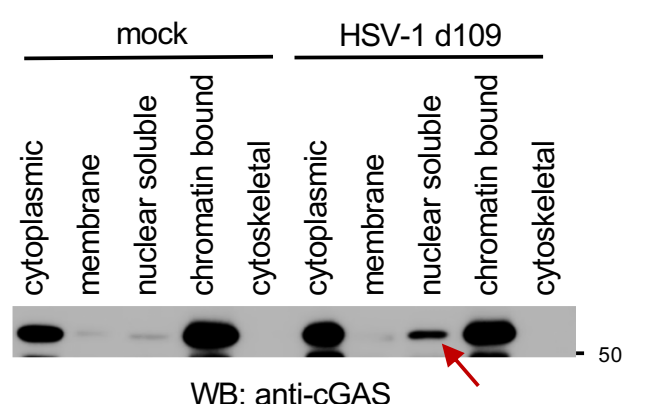

WB: anti-cGAS

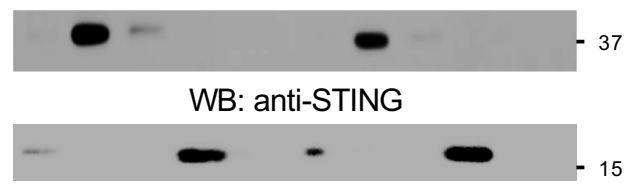

WB: anti-histone $\mathrm{H} 3$

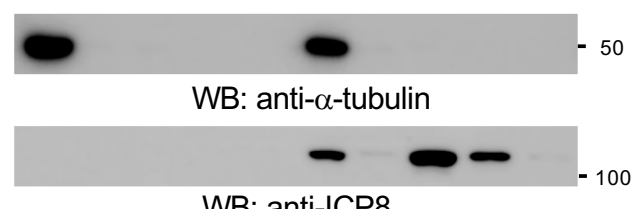

WB: anti-ICP8

E
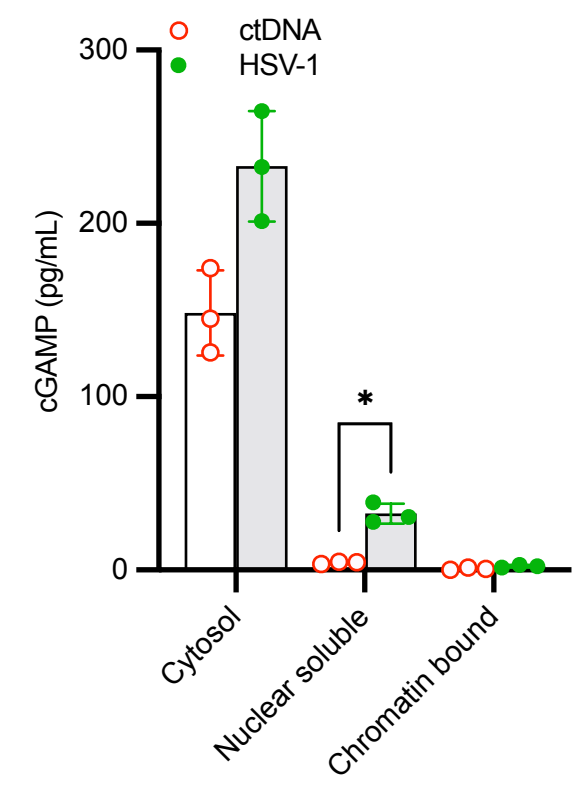

HSV-1
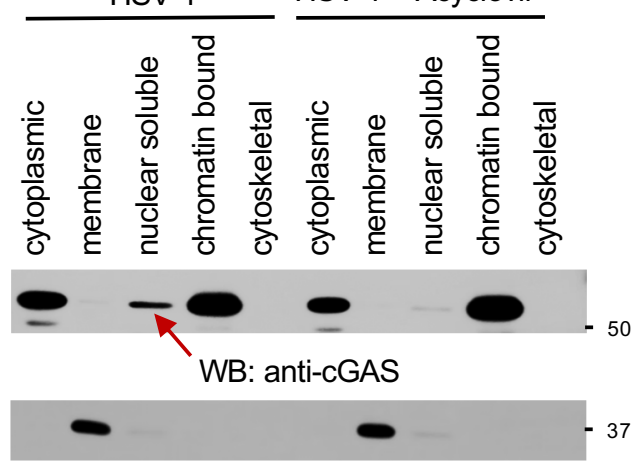

WB: anti-STING

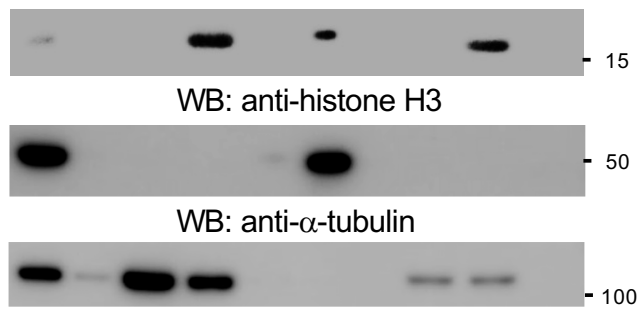

WB: anti-ICP8

F

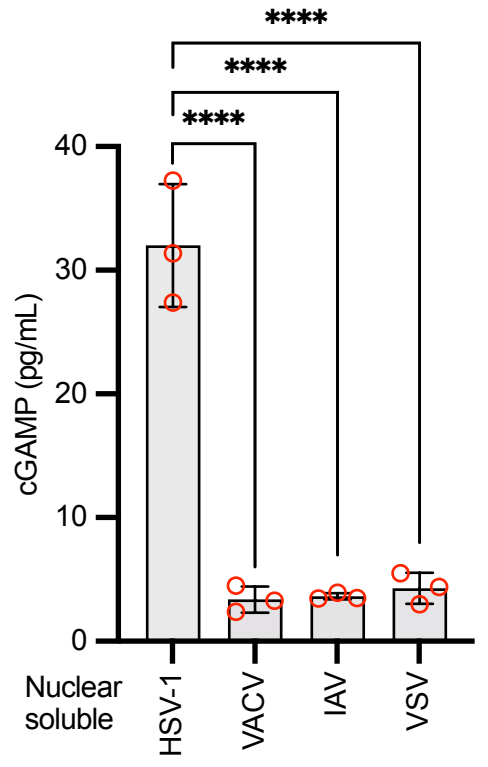

Figure 2 
bioRxiv preprint doi: https://doi.org/10.1101/2021.08.27.457948; this version posted August 28, 2021. The copyright holder for this preprint (which was not certified by peer review) is the author/funder. All rights reserved. No reuse allowed without permission.

A

B

CGAS

D

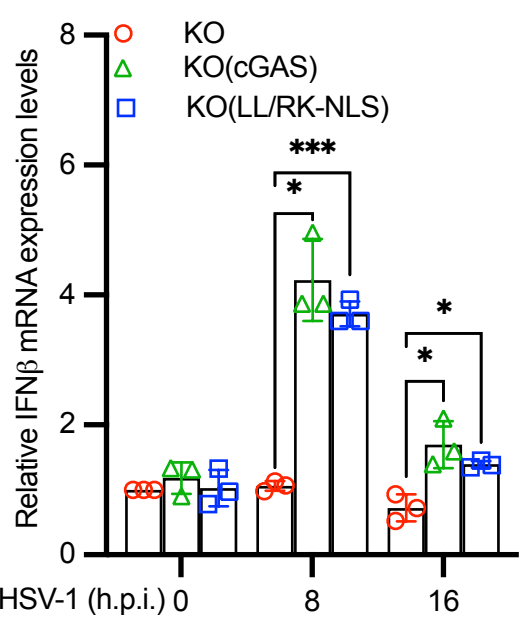

E

\begin{tabular}{|c|c|c|c|c|c|c|c|c|c|}
\hline \multirow[b]{2}{*}{ HSV-1 (h) } & \multicolumn{3}{|c|}{$\mathrm{KO}$} & \multicolumn{3}{|c|}{$\mathrm{KO}(\mathrm{cGAS})$} & \multicolumn{3}{|c|}{$\mathrm{KO}(\mathrm{LL} / \mathrm{RK}-\mathrm{NLS})$} \\
\hline & 0 & 4 & 8 & 0 & 4 & 8 & 0 & 4 & 8 \\
\hline$\pi$ & 0.05 & 0.05 & 0.05 & 0 & 1.71 & 0.42 & 0 & 2.2 & 1.1 \\
\hline
\end{tabular}

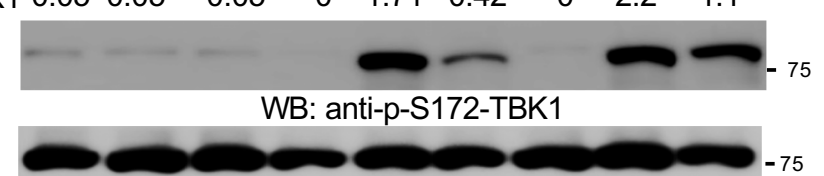

WB: anti-TBK1

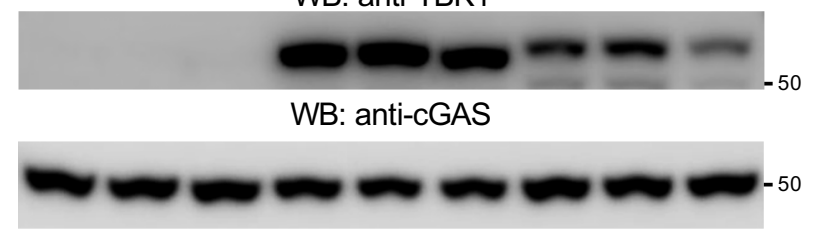

WB: anti- $\alpha$-tubulin
RAW 264.7 cells

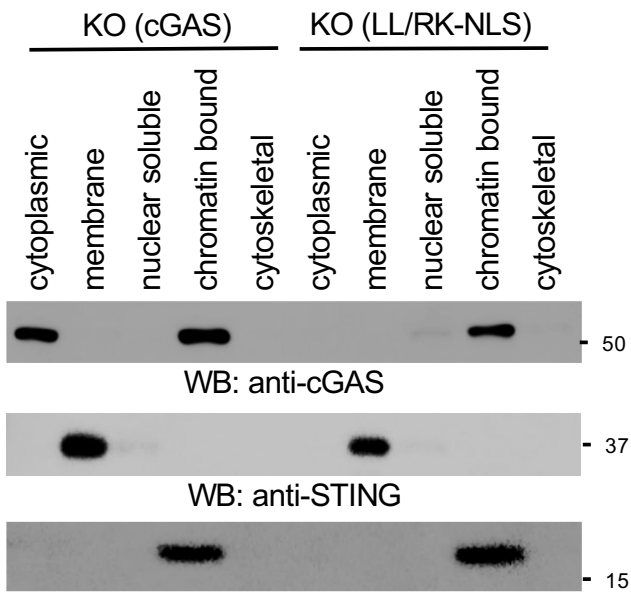

WB: anti-histone $\mathrm{H} 3$

WB: anti-tubulin

C

cGAS KO (LL/RK-NLS)

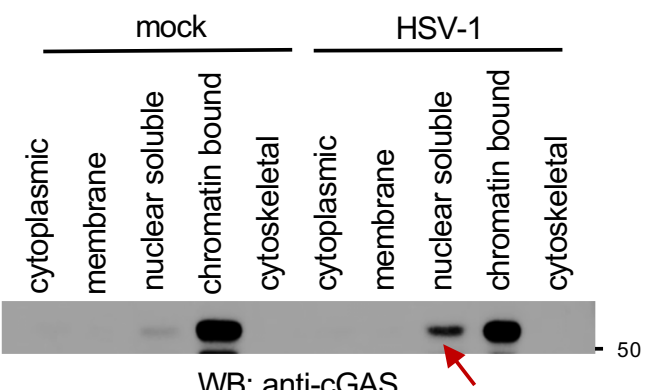

WB: anti-cGAS

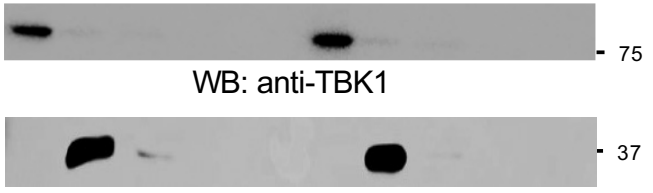

WB: anti-STING

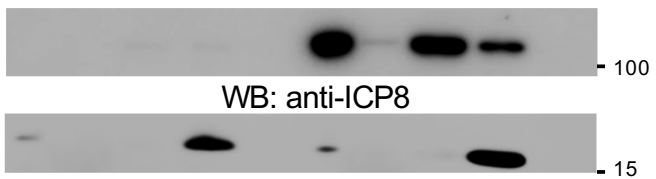

WB: anti-histone H3

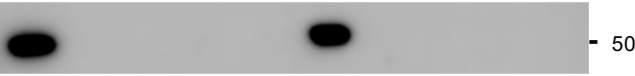

WB: anti-tubulin

$\mathrm{F}$

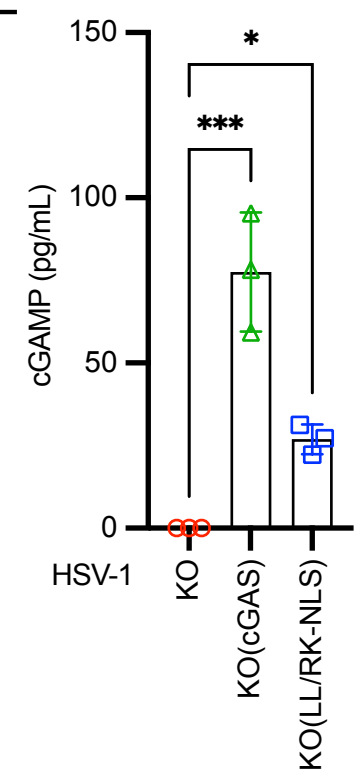

G

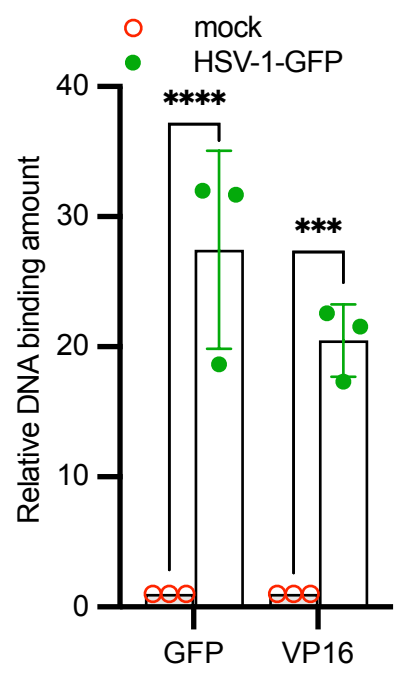

Figure 3 
A

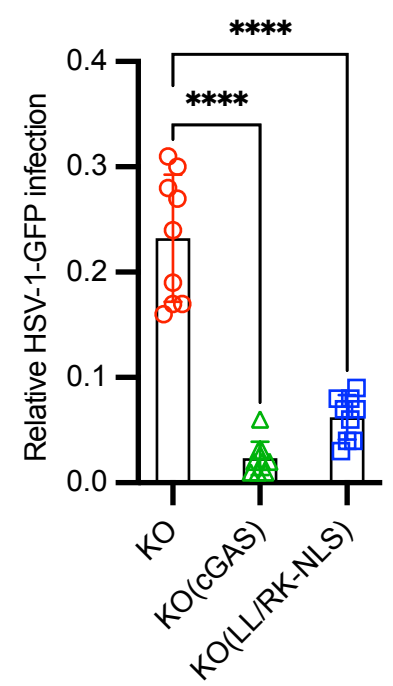

D

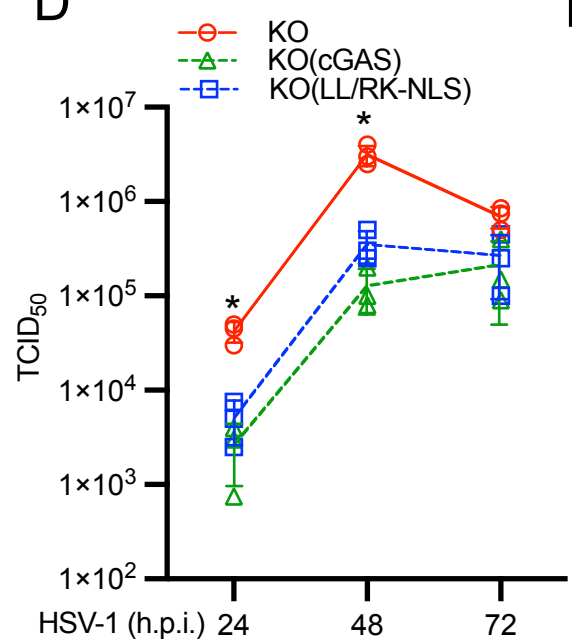

B

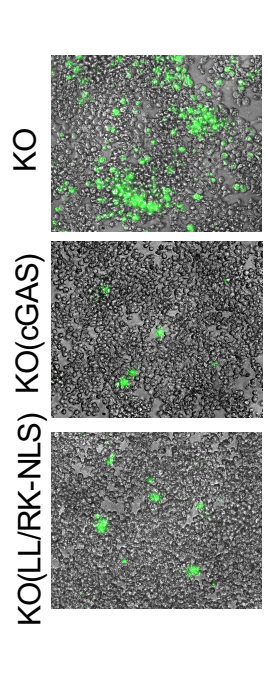

E

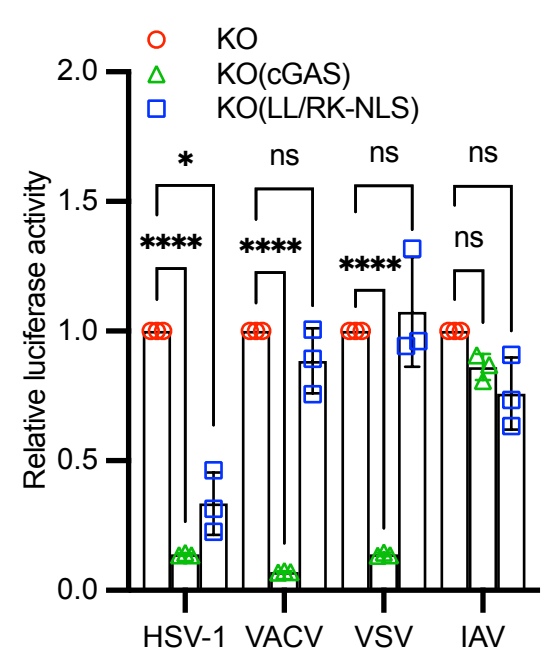

C

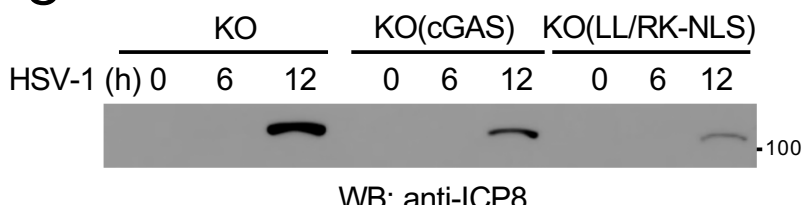

WB: anti-ICP8

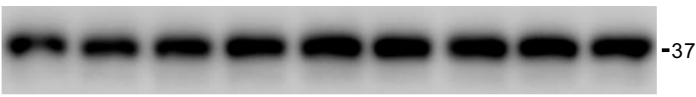

WB: anti-STING

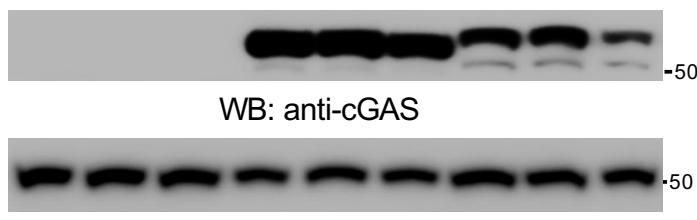

WB: anti- $\alpha$-tubulin

F

Nucleosome $\mathbf{X}$ Inactive cGAS

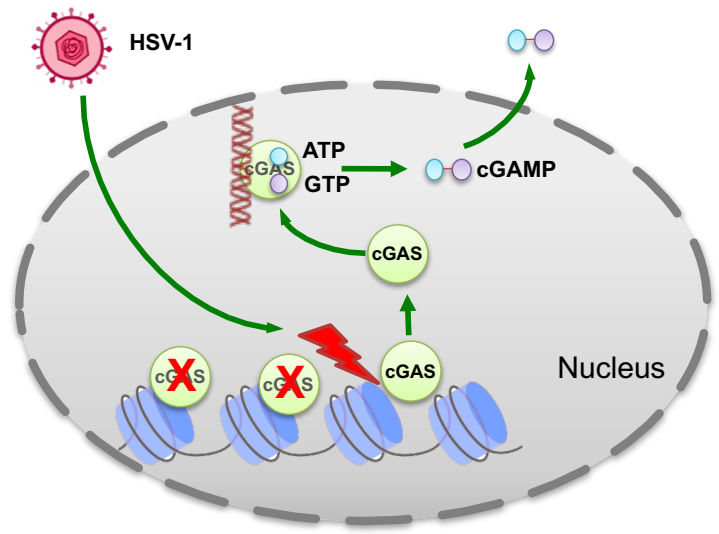

Figure 4 